\title{
The pre-partum, low-frequency, sinusoidal rhythm due to placental abruption-A case study ${ }^{*}$
}

\author{
Shaohua Liu, Jinbo Zhao, Dongxia Yang", Ru Zhang, Hui Wang \\ Department of Gynecology and Obstetrics, Yantai Economic and Technological Development Zone Hospital, Shandong, China \\ Email: ${ }^{*}$ mcydx@163.com
}

Received 26 January 2013; revised 28 February 2013; accepted 9 March 2013

\begin{abstract}
We consider that a low-frequency, sinusoidal rhythm detected by Electronic Fetal Monitoring (EFM) is a typical manifestation of a placental abruption. We present the case of a 26-year-old woman who was gestational 36 weeks. She had irregular contractions. The EFM showed a low-frequency, sinusoidal rhythm. The cycle of the sine curve increased in time from 90 seconds to 160 seconds gradually. A severely asphyxiated newborn was successfully resuscitated after an emergency Cesarean Section. The mother and child were discharged from our hospital in a good general condition seven days later. Upon examination it was proven that this was a case of a placental abruption. This is an original case study report about how to diagnose a placental abruption according to an EFM reading. We propose a definition of a low-frequency, sinusoidal rhythm having: 1) a stable baseline FHR (fetal heart rate) of 120 - $160 \mathrm{bpm}$; 2) a possible variation of 20 - $30 \mathrm{bpm}$; 3) a frequency of 90 - $160 \mathrm{sec}-$ onds per cycle; 4) fixed or flat short-term variability; 5) oscillation of the sinusoidal wave from above and below a baseline; 6) no areas of normal FHR variability or reactivity. What is more, the lengths of the fluctuation cycle and the greater the amplitude has a close relationship with the prognosis of both mother and child. This pre-partum, low-frequency, sinusoidal rhythm is an ominous sign of fetal jeopardy needing immediate intervention. The medical literature has never reported such an association. Therefore, our case study report is possibly the first to mention this observation.
\end{abstract}

Keywords: Placental Abruption; Pre-Partum; Low-Frequency; Sinusoidal Rhythm

\section{INTRODUCTION}

Placental abruption means a separation of the placenta

\footnotetext{
*The authors have no competing interests hereby to declare.

No funds were granted to support the present study.

${ }^{\#}$ Corresponding author.
}

from its normal implantation after 20 weeks of gestation, or during the course of delivery. Placental abruption can seriously harm both the mother and the child. Its rate of occurrence is $0.46 \%-2.1 \%$ [1]. The perinatal mortality rate is about $20.0 \%-42.8 \%$ [1]. We can diagnose placental abruption according to clinical symptoms and ultra-sonography, but it is hard to make a definite diagnosis due to the mild symptoms. The accuracy of color, Doppler ultra-sonography in diagnosis of abruption is $52 \%$ [2]. We found that maybe the EFM is a good method to diagnose it. Pre-partum EFM of this gravida showed that there emerged a sinusoidal curve, but it was not a typical high-frequency sinusoidal curve.

Modanlou and Murata [3] proposed the following definition for the interpretation of true sinusoidal rhythm as: 1) a stable baseline FHR (fetal heart rate) of 120 $160 \mathrm{bpm}$; 2) a variation of 5 - $15 \mathrm{bpm}$, rarely greater; 3) a frequency of $2-5$ cycles per minute; 4) a fixed or flat short-term variability; 5) an oscillation of the sinusoidal wave from above and below a baseline; 6) no areas of normal FHR variability or reactivity. The sinusoidal rhythm is a rare occurrence [4]. Its incidence is $0.41 \%$ (8/19506), 62.5\% (5/8) accompanied by neonatal anemia (hemoglobulin $<10 \mathrm{~g} / \mathrm{dL}$ ).

However, according to Cao Zeyi [5] the sinusoidal rhythm should be divided into two types; the high-frequency sinusoidal rhythm and the low-frequency sinusoidal rhythm. The high-frequency sinusoidal rhythm is equivalent to the true sinusoidal rhythm; it is always seen in RH blood group incompatibility. The low-frequency sinusoidal rhythm means a frequency of 2 - 5 minutes per cycle. This is rare but is associated with severe, fetal hypoxia and needs immediate intervention [6,7]. In our observation, this atypical sinusoidal curve is a low-frequency sinusoidal curve. It is a typical manifestation of a placental abruption.

\section{THE CASE}

A 26-year-old primigravida was admitted to our department at 36 weeks of gestation, based on her last monthly period, due to intermittent lower abdominal pains which 
had started the day before. The course of her pregnancy was generally uneventful and a standard fetal ultrasound assessment was carried out. Upon abdominal examination the fundal height was consistent with 36 weeks of gestation and vertex presentation. A trans-abdominal ultrasound scan revealed a viable fetus with biometry consistent with 36 weeks of gestation and an estimated fetal weight of $2.500 \mathrm{~kg}$ with no visible morphological anomalies. The maximum vertical pocket depth was $7 \mathrm{~cm}$, the placenta was grade II, and there was no evidence of any retro-placental clot.

However, the EFM was found to be abnormal (Figure 1). The pre-partum EFM in this case showed that there emerged an atypical, low-frequency, sinusoidal curve. The baseline FHR was $135 \mathrm{bpm}$, and there was no fetal movement (FM) during the monitoring. The monitoring time was prolonged but there still was no FM. The FHR baseline oscillation was very regular; it waved above and below a baseline just like the sine curve. Its variation was about $30 \mathrm{bpm}$, and the swing period was about $90 \mathrm{sec}-$ onds. It had a fixed, short-term variability and no area of normal FHR variability or reactivity. The gravida felt that the FM was as normal as before, so we could not distinguish a true sine curve from pseudo ones. After dealing with hyperventilation, the gravida was given oxygen and left in a lying position. A second EFM was conducted (Figure 2). There was only one FM cycle without the corresponding FHR speeding up. The baseline FHR was $155 \mathrm{bpm}$, its variation was $30 \mathrm{bpm}$. The FHR baseline oscillation was still very regular, and the swing cycle lasted about 140 seconds. It was obvious to us that this was not a true sinusoidal rhythm i.e. it was not a high-frequency, sinusoidal rhythm. As a team, we agreed that this was indicative of a low-frequency sine

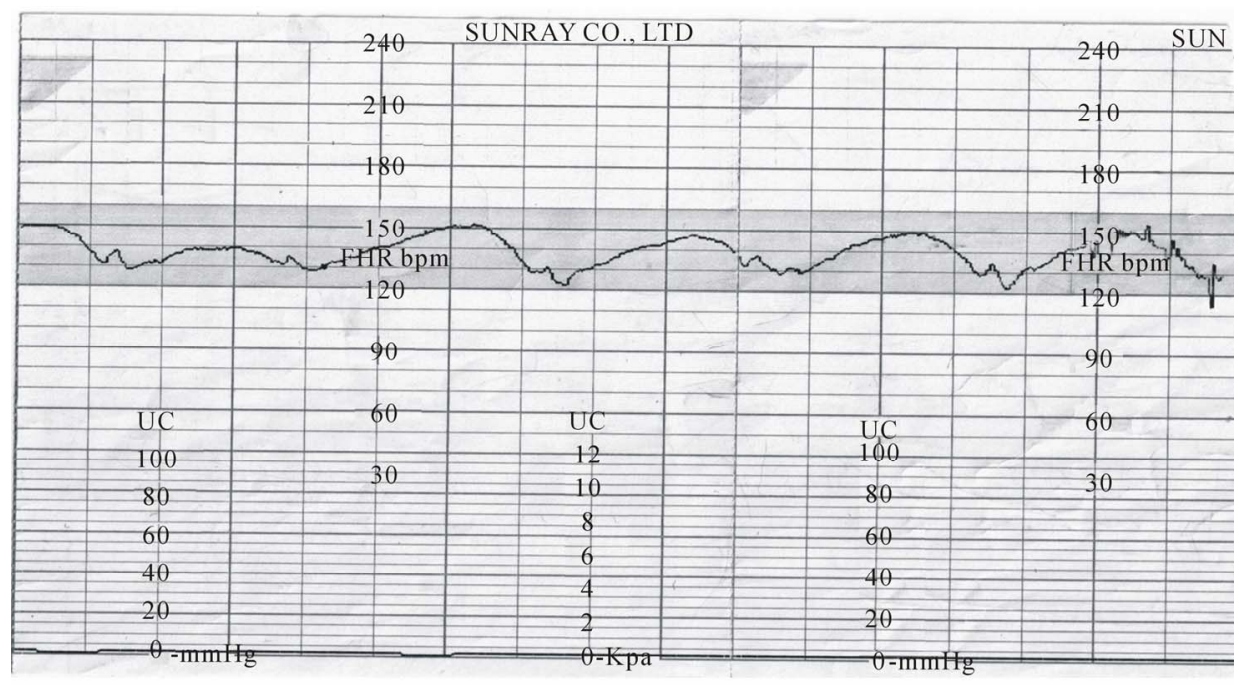

Figure 1. One fluctuation cycle is about 90 seconds (velocity is $2 \mathrm{~cm} / \mathrm{min}$ ).

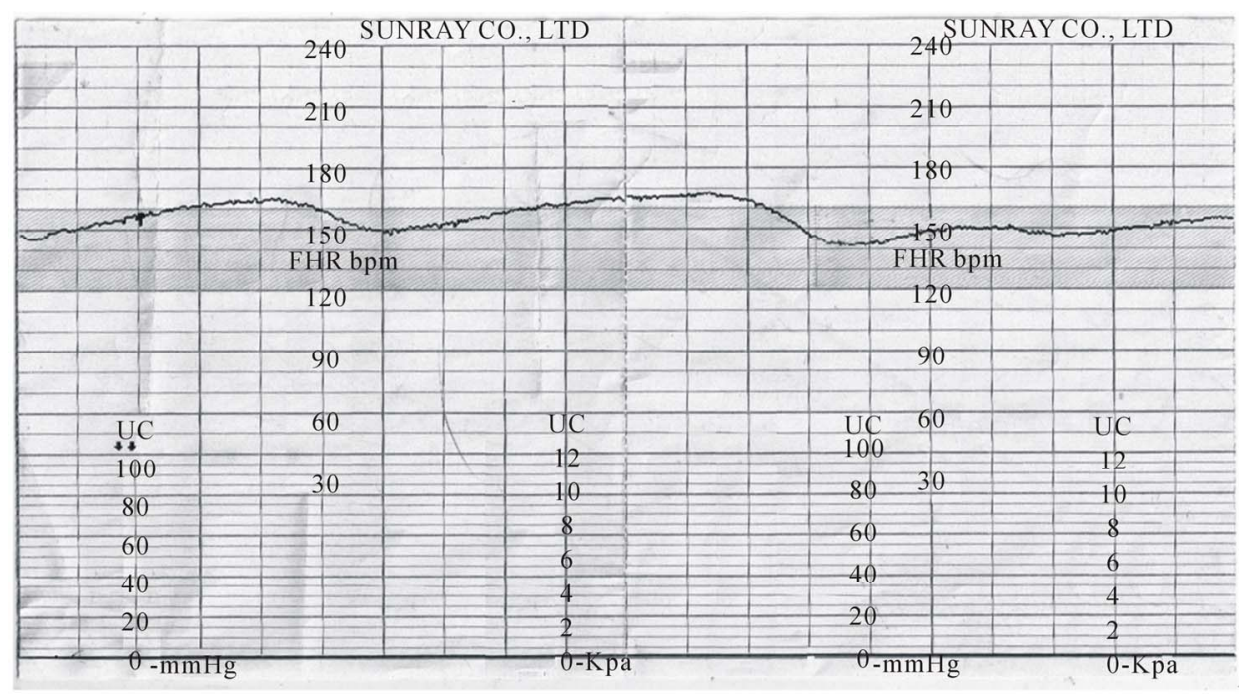

Figure 2. One fluctuation cycle increased to 140 seconds (velocity is $3 \mathrm{~cm} / \mathrm{min}$ ). 
curve and that the fetus was in imminent danger in uterus, so a Cesarean Section (CS) was recommended to rescue the fetus. The gravida and her husband were worried about the preterm fetus and they insisted on waiting for several days. They preferred to try a normal, vaginal delivery. Continuous EFM showed that the cycle of the sine curve increased to 160 seconds gradually and its variation was 20 bpm (Figure 3). We communicated with the couple over and over again that it was very important to do CS immediately or that the fetus might die. Although the fetus was preterm, it was estimated to be very much alive. Finally, the couple consented to a CS. A hema-glutination inspection showed that the fibrinogen level was $1.97 \mathrm{~g} / \mathrm{L}$, so blood was prepared in case a blood transfusion was needed. The patient's hemoglobin dropped from normal to $70 \mathrm{~g} / \mathrm{L}$ after the operation. After careful preparation, an emergency CS was performed.

A laparotomy was undertaken through a low-transverse incision. When the peritoneum was cut, there was about $50 \mathrm{ml}$ of old blood ascites. The uterus was opened by a low-transverse section. The amniotic fluid was clear; its volume was about $1500 \mathrm{ml}$. A premature, baby boy weighing $2.500 \mathrm{~kg}$ with no obvious abnormalities was delivered. Effective neonatal cardiopulmonary resuscitation was carried out immediately. Apgar scores of 3, 6 and 10 were recorded at the 1 st, 5 th and 10 th minutes of life. There was much clotted blood expelled from the uterine cavity after the placenta separated from the uterus. Blood loss was estimated to be about $1000 \mathrm{ml}$. The uterus and accessories were detected during the operation. There was a violet, streak ecchymosis on the left corneal, so the placenta was examined carefully. It was about 10 $\mathrm{cm} \times 10 \mathrm{~cm} \times 2 \mathrm{~cm}$ and it connected with another likesized accessory placenta. There was obvious coagulation clot pressure on the accessory placenta, causing a visible slash-like mark which was about $10 \mathrm{~cm} \times 5 \mathrm{~cm}$. This definitely confirmed that the diagnosis of a placental abruption was correct.

Post-operative observations showed old blood ascites and the utero-placental hemorrhaging hint that the blood pressure between the placenta and the uterus inner wall is too severe that the blood penetrated the myometrium. In this instance the amniotic fluid was clear. Studies show that in cases of placental abruption, bloody amniotic fluid is present in only about $15 \%$ of cases [7].

The low blood fibrinogen showed that the patient's coagulation system was started to be destroyed. Although the patient's blood loss was $1000 \mathrm{ml}$, the patient was safe because of the timely operation, and she didn't need a blood transfusion. The postpartum hemorrhagic rate is about $18.85 \%$ - $32.06 \%$ in cases of placental abruption [8], and in these cases the patients almost always need a blood transfusion. In this case the newborn with severe asphyxia was rescued successfully. Both the mother and child recovered fully. We believe that the key to this result was our recognition of the low-frequency, sinusoidal rhythm EFM, and the timely and decisive Csection operation.

On the 7th postoperative day, the mother and her child were both discharged in good condition.

\section{DISCUSSION}

We consider that the pre-partum, low-frequency, sinusoidal rhythm is a typical manifestation of a placental abruption. A placental abruption signifies a serious and potentially fatal complication for both the mother and child. But it is difficult to diagnose a placental abruption due to the mild signs and symptoms. An ultrasound inspection can be helpful but it is not always effective in these cases but we believe that EFM has tremendous guiding significance for cases of placental abruption. We now consider that the pre-partum, low-frequency, sinusoidal rhythm to be a typical indicator of placental abruption. What is more, both the length of the fluctuation cycle and the variations have a close relationship with the prognosis of both the mother and the child. Long fluctuation cycles with significant variations appear to indicate a dangerous situation for the fetus.

Therefore, we propose a definition for the low-frequency, sinusoidal rhythm to suspect cases of placental abruption and fetal jeopardy. We recognize an EFM,

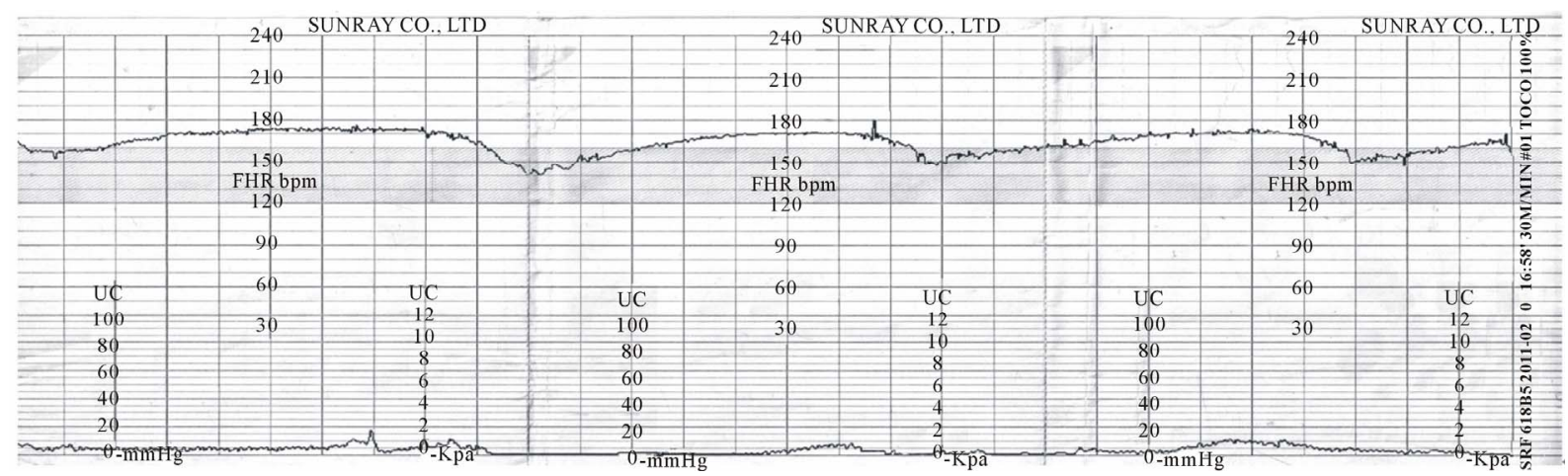

Figure 3. One fluctuation cycle increased to 160 seconds (velocity is $3 \mathrm{~cm} / \mathrm{min}$ ). 
low-frequency, sinusoidal rhythm as: 1) a stable baseline FHR of 120 - $160 \mathrm{bpm}$; 2) a potential variation of 20 - 30 bpm; 3) a frequency of 90 - 160 seconds per cycle; 4) a fixed or flat short-term variability; 5) an oscillation of the sinusoidal wave from above and below a baseline; 6) no areas of normal FHR variability or reactivity. We consider this low-frequency, sinusoidal curve to be related to the worst possible neonatal outcome and needs immediate, emergency, medical intervention. Still, more cases are needed to verify the characteristics of this low-frequency, sinusoidal curve to undisputedly show its importance on diagnosing cases of placental disruption.

\section{CONCLUSION}

Medical professionals recognizing a low-frequency, sinusoidal rhythm on EFM should immediately suspect fetal jeopardy, probably due to placental abruption.

\section{CONSENT}

Written informed consent was obtained from our patient for publication of this case study and for accompanying images. A copy of this written consent is available for review by editors-in-chief of any interested journal.

\section{AUTHOR'S CONTRIBUTIONS}

L SH wrote the paper, checked the medical records and the literature, $\mathrm{Z}$ JB revised the manuscript in accordance with the reviewers suggestions. $\mathrm{L} \mathrm{SH}$ and $\mathrm{WH}$ were the surgeons who performed the operation. Y DX guided us to write the paper. ZR performed the monitoring sections. All authors have read and approved the final manuscript.

\section{REFERENCES}

[1] Cao, Z.Y. (2010) Obstetrics and gynecology. Clinical Edition, People's Medical Publishing House, Beijing, 145.

[2] Masselli, G., Brunelli, R., Di Tola, M. et al. (2011) MR imaging in the valuation of placental abruption: Correlation with sonographic findings. Radiology, Epub Ahead of Print. doi:10.1148/radiol.10101547

[3] Modanlou, H.D. and Murata, Y. (2004) Sinusoidal heart rate pattern: Reappraisal of its definition and clinical significance. Journal of Obstetrics and Gynaecology Research, 30, 169-180. doi:10.1111/j.1447-0756.2004.00186.x

[4] Reddy, A., Moulden, M. and Redman, C.W. (2009) Antepartum high-frequency fetal heart rate sinusoidal rhythm: Computerized detection and fetal anemia. American Journal of Obstetrics \& Gynecology, 200, e1-e6.

[5] Cao, Z.Y. (2010) Obstetrics and gynecology. Clinical Edition, People's Medical Publishing House, Beijing, p. 46.

[6] Kayani, S.I., Walkinshaw, S.A. and Preston, C. (2003) Pregnancy outcome in severe placental abruption. BJOG, 110, 679-683. doi:10.1046/j.1471-0528.2003.02088.x

[7] Wang, Y.N. and Yang, Z. (2010) Multivariate analysis of pregnancy outcomes and investigation of precaution in placental abruption. Chinese Journal of Obstetrics \& Gynecology and Pediatrics, 26, 114-118.

[8] (2003) Shanghai’s investigating team on placental abruption. The study on Shanghai's placental abruption in 10 years. Shanghai Medicine, 26, 137-139.

\author{
ABBREVIATIONS \\ EFM: Electronic Fetal Monitoring \\ FM: Fetal Movement \\ FHR: Fetal Heart Rate \\ CS: Cesarean Section
}

